\title{
Esophageal Perforation: A Rare Complication of Transesophageal Echocardiography in a Patient with Asymptomatic Esophagitis
}

\author{
Kabir Ahmed ${ }^{\mathrm{a}}$ Yasir Lal $^{\mathrm{b}}$ Steven Condron ${ }^{\mathrm{c}}$ \\ ${ }^{a}$ Division of Internal Medicine, Sanford School of Medicine, University of \\ South Dakota, and ${ }^{b}$ Division of Internal Medicine and ${ }^{\mathrm{c}}$ Division of \\ Gastroenterology, Avera McKennan Hospital \& University Health Center, \\ Sioux Falls, S. Dak., USA
}

\section{Key Words}

Esophageal perforation · Transesophageal echocardiography · Esophagitis · Esophageal stenting

\begin{abstract}
Transesophageal echocardiography (TEE) is a commonly used procedure in patients with suspected endocarditis. A rare but dreadful complication of this procedure is perforation of the esophagus. We report the case of an elderly female with multiple comorbidities, who presented with polyarticular septic arthritis. TEE was performed to rule out endocarditis. Though the standard procedure protocol was followed, she developed esophageal perforation. It was managed with esophageal stenting but she developed multiorgan failure and did not survive. This case highlights the potential of severe morbidity and mortality associated with TEE. Appropriate screening must be done and high-risk individuals must be identified before such procedures are attempted.
\end{abstract}

\section{Introduction}

Transesophageal echocardiography (TEE) is a valuable diagnostic tool that is used routinely to diagnose endocarditis in appropriate clinical settings. It is considered relatively safe and complications are uncommon. One of the most serious but rare complication of TEE is perforation of the esophagus. Such perforations continue to have high morbidity and mortality rates, with fearsome outcomes of mediastinitis, sepsis and multiorgan failure [1]. In a large series of 10,000 cases of TEE, esophageal perforation was reported in 3 cases $(0.03 \%)$ with no mortalities [2]. We present a 
unique case of esophageal perforation in which the patient was asymptomatic, with no signs of esophageal pathology in her history and on physical examination. TEE was performed following standard protocols to rule out endocarditis, yet the patient developed esophageal perforation and died from its complications.

\section{Case Report}

An 87-year-old Caucasian female presented with signs and symptoms of left knee septic arthritis and bilateral conjunctivitis. She additionally had swelling in one of her hand joints and in the first metatarsophalangeal joint of her left foot. Her symptoms included fevers and episodic altered mental status from the past 4 days. She denied any sore throat, dysphagia, odynophagia, hoarseness or other esophageal symptoms. Her past medical history included hypertension, paroxysmal atrial fibrillation, diabetes mellitus type I, hypothyroidism, sick sinus syndrome status post pacemaker placement with no indications of any esophageal abnormalities. Her past surgical history included total abdominal hysterectomy, multiple caesarean deliveries, cholecystectomy and hernia repair. On physical examination, the patient was in no acute distress; eye examination revealed bilateral conjunctival erythema and mild mucopurulent discharge. Her heart rhythm was noted to be irregular. Her left knee was swollen, erythematous and tender on passive extension and flexion. Laboratory examination showed elevated white blood cell count $(14,600 / \mu \mathrm{l}$, normal $3,300-10,900 / \mu \mathrm{l})$, high erythrocyte sedimentation rate $(30 \mathrm{~mm} / \mathrm{h}$, normal $0-22 \mathrm{~mm} / \mathrm{h})$ and elevated C-reactive protein (187.7 mg/l, normal 0.0-9.9 mg/l).

To rule out endocarditis, TEE was performed after inconclusive transthoracic echocardiography (TTE). After intravenous sedation with midazolam and pharyngeal local anesthesia with benzocaine (20\%), the standard protocol of introducing the esophageal transducer blindly was followed and diagnostic images were obtained. Immediately after the procedure, the patient was in moderate distress, with symptoms of nausea, vomiting and severe chest and back pain. Urgent chest radiography and Gastrografin study was performed which showed an upper third esophageal perforation; contrast was seen immediately above the gastroesophageal junction (fig. 1). An urgent esophagogastroduodenoscopy (EGD) was performed which confirmed the mucosal tear and additionally showed moderate to severe inflammation throughout the esophagus with no obvious bleeding (fig. 2). A WallFlex stent (Boston Scientific, Natick, Mass., USA) was placed to cover the esophageal tear (fig. 3). The patient was transferred to the intensive care unit, and Diflucan and broad-spectrum antibiotics were started. A repeat Gastrografin esophagram after 2 days did not show any leakage and a liquid diet was started. However, the patient developed respiratory failure followed by multiorgan failure. Due to advanced age and multiple comorbidities, the family decided to proceed with palliative care only and the patient expired after 1 week

\section{Discussion}

TEE is mostly performed when TTE is inadequate in appropriate clinical settings. Other indications include infective endocarditis, native and prosthetic valvular disease, systemic embolization, intraoperative monitoring, congenital heart condition, ischemic heart disorder, and pathology of atrium, interatrial septal and thoracic aorta [3]. Our patient presented with polyarticular septic arthritis. TTE did not show any obvious vegetation and therefore TEE was performed to investigate the source of infection.

TEE-induced perforations are perceptively catalyzed by intolerable mucosal pressure and thermal damage effectuated by the probe, and/or from pre-existing esophageal abnormalities [2,4]. Contraindications to TEE include substantial dysphagia, severe instability of cervical vertebrae and esophageal disorders such as diverticulum, fistula, laceration, severe inflammation, stricture, tumor and varices [3]. Other perforation-cajoling factors may include advanced age and operator inexperience 
[1]. As highlighted in the case, asymptomatic severe esophagitis and advanced age may have contributed to perforation in our patient.

Medical instrumental manipulation is the most common cause of esophageal perforation, with cervical esophagus vicinity being the most common site [4, 5]. Other causations include trauma, tumor, foreign body ingestion, caustic injury, pill-induced and vehement vomiting, etc. Classical esophageal perforation symptoms consist of chest pain, dyspnea, emesis, fever and subcutaneous emphysema. The diagnosis is usually made by an immediate upright chest X-ray followed by Gastrografin esophagram. In some cases, chest CT may be beneficial [5].

Surgical closure of the perforation has traditionally defined the treatment of esophageal perforation. Recently, conservative medical management has gained popularity, which compromises broad-spectrum intravenous antibiotics, parenteral nutrition, nothing by mouth, nasogastric suction and possible use of mediastinoscopy [5]. In the past several decades, placing an esophageal endoscopic stent has prevailed as a characteristic feature of the nonoperative approach. In a small study of 17 patients, endoluminal stents successfully occluded $94 \%$ of esophageal leaks [6]. Another small study showed that stent placement provided rapid closure of these perforations with less morbidity, while avoiding surgical approaches such as thoracotomy or celiotomy [7]. Particular to our case, a silicone-coated WallFlex stent was used, with flexible braided construction and thick nitinol wires. Unfortunately, the overall mortality rate of esophageal perforation continues to be high, ranging from 5 to 63\% [5]. Furthermore, there are limited data available on esophageal stent benefits, risks and complications as compared to the surgical approach, and further extensive studies are needed.

In conclusion, TEE is a commonly used diagnostic procedure that is considered safe with relatively few complications. Our case highlights a significant and potentially fatal complication of TEE in asymptomatic esophagitis. It is absolutely crucial to identify such high-risk individuals prior to the procedure and to assess for risks and benefits before the procedure is attempted.

\section{Disclosure Statement}

The authors state no conflict of interest. 


\begin{tabular}{r|l|l|l}
$\begin{array}{r}\text { Case Reports in } \\
\text { Gastroenterology }\end{array}$ & $\begin{array}{l}\text { Case Rep Gastroenterol 2012;6:760-764 } \\
\text { DOI: 10.1159/000346311 }\end{array}$ & $\begin{array}{l}\text { Published online: } \\
\text { December 20, 2012 }\end{array}$ & $\begin{array}{l}\text { @ 2012 S. Karger AG, Basel } \\
\text { ISSN 1662-0631 } \\
\text { www.karger.com/crg }\end{array}$ \\
\hline
\end{tabular}

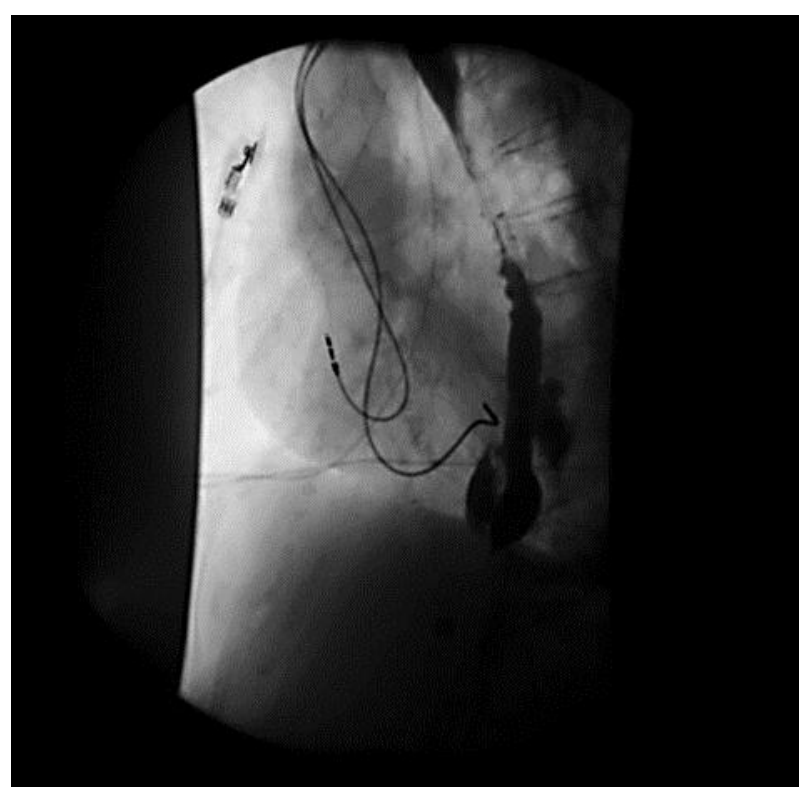

Fig. 1. Gastrografin esophagram showing contrast extravasation immediately above the gastroesophageal junction.

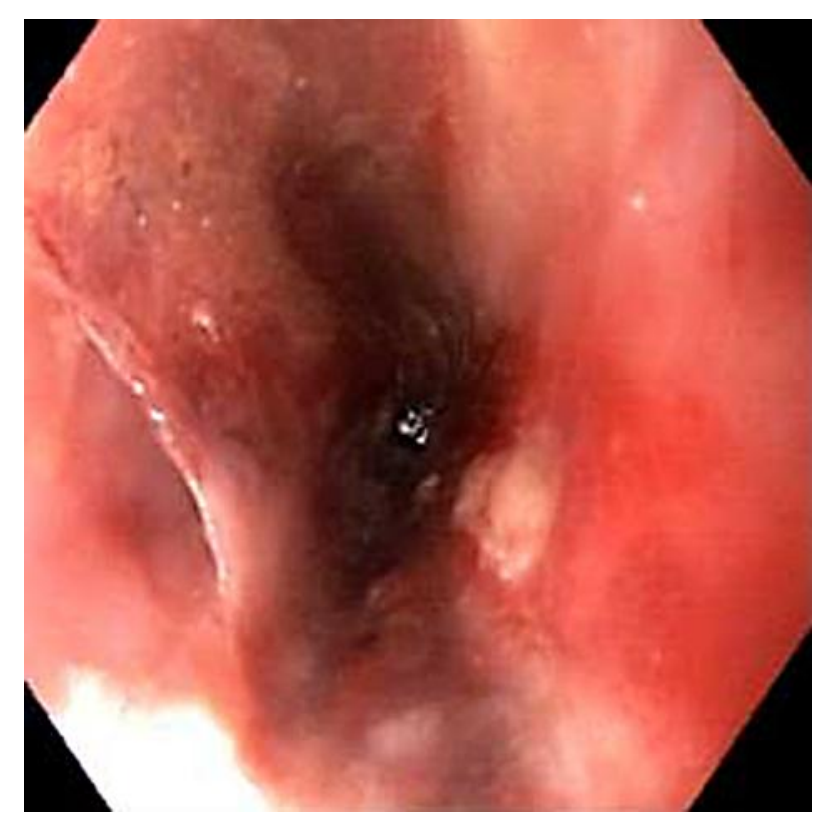

Fig. 2. EGD showing severe esophagitis along with mucosal tear. 


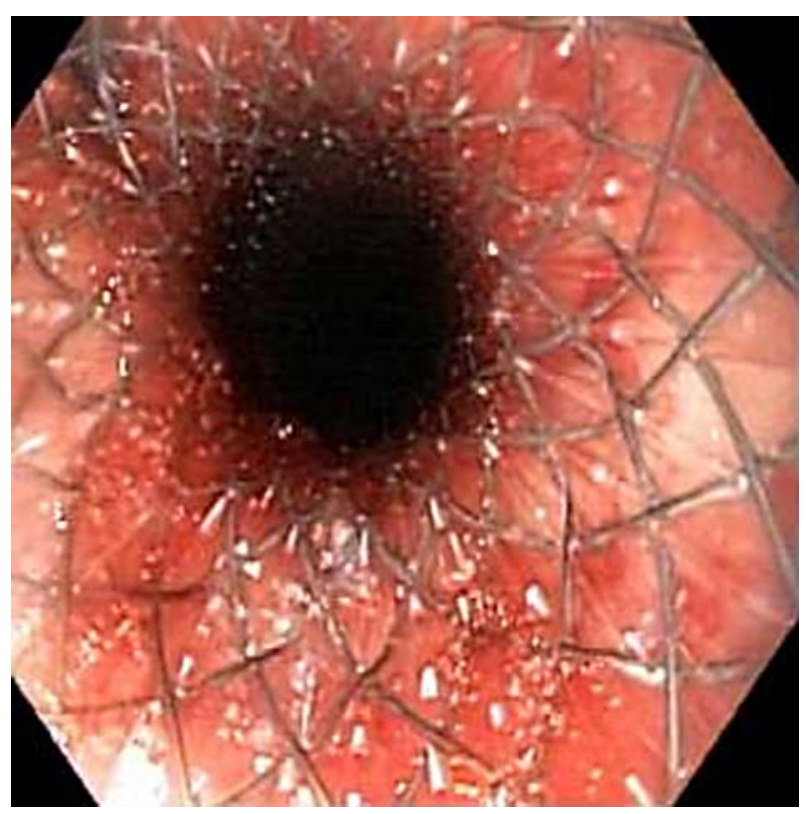

Fig. 3. Endoscopic view of stent placement covering the mucosal tear shown in figure 2.

\section{References}

1 Sobrino MA, Kozarek R, Low DE: Primary endoscopic management of esophageal perforation following transesophageal echocardiogram. J Clin Gastroenterol 2004;38:581-585.

$\checkmark 2$ Min JK, Spencer KT, Furlong KT, DeCara JM, Sugeng L, Ward RP, Lang RM: Clinical features of complications from transesophageal echocardiography: a single-center case series of 10,000 consecutive examinations. J Am Soc Echocardiogr 2005;18:925-929.

3 Gring C, Griffin BP: Transesophageal echocardiography; in Topol EJ (ed): Textbook of Cardiovascular Medicine, ed 3. New York, Lippincott Williams \& Wilkins, 2006, pp 868-871.

4 Massey SR, Pitsis A, Mehta D, Callaway M: Oesophageal perforation following perioperative transoesophageal echocardiography. Br J Anaesth 2000;84:643-646.

5 Infantino A, Ter RB: Rupture and perforation of the esophagus; in Castell DO, Richter JE (eds): The Esophagus, ed 4. New York, Lippincott Williams \& Wilkins, 2003, pp 648-657.

6 Freeman RK, Van Woerkom JM, Ascioti AJ: Esophageal stent placement for the treatment of iatrogenic intrathoracic esophageal perforation. Ann Thorac Surg 2007;83:2003-2007.

7 Freeman RK, Van Woerkom JM, Vyverberg A, Ascioti AJ: Esophageal stent placement for the treatment of spontaneous esophageal perforations. Ann Thorac Surg 2009;88:194-198. 